\title{
Alterssicherung und Politik bei schrumpfender Bevölkerung
}

In Deutschland (zusammengerechnet Ost- und Westdeutschland) sterben seit 1972 mehr Menschen als geboren werden. Die Geburtenzahl hat sich seit dem stärksten Geburtenjahrgang 1964 mit 1,4 Mill. Geburten auf 2013 unter 700000 halbiert. Ohne die Zuwanderung (auch aus Polen) wäre die deutsche Bevölkerung bereits um mehr als 5 Millionen Menschen geschrumpft und würde nicht 82 Mill., sondern weniger als 77 Mill. betragen. In den nächsten Jahrzehnten bis 2050 würde die deutsche Bevölkerung ohne Zuwanderung auf 62 Mill. schrumpfen. Bereits heute hat Frankreich bei 64,3 Mill. Einwohnern eine höhere jährliche Geburtenzahl als Deutschland. ${ }^{2}$ Frankreich könnte also die deutsche Bevölkerungszahl übertreffen, ein altes französisches Anliegen. Nicht zuletzt um seinem „Erzfeind Deutschland“ in der Truppenstärke nicht unterlegen zu sein, hatte Frankreich früh eine aktive Bevölkerungs- / Familienpolitik eingeführt. In Deutschland beträgt die Geburtenrate pro Frau 1,36 Kinder.

1 Joachim Wiemeyer - Prof. Dr. lic. theol. Promotion in Volkswirtschaftslehre, Habilitation für Christliche Sozialwissenschaften an der Kath. Theol. Fakultät der Universität Münster. Seit 1998 Prof. für Christliche Gesellschaftslehre an der Ruhr-Universität in Bochum, Berater der Caritaskommission der Deutschen Bischofskonferenz. Mitglied der Sachverständigenkommission „Weltwirtschaft und Sozialethik“ der Kommission Weltkirche der Deutschen Bischofskonferenz. Mitglied im Ethikbeirat der Pax-Bank. Forschungsschwerpunkte: Wirtschaftsethik, Politische Ethik, Wohlfahrtsverbände und Sozialpolitik.

2 Vgl.T. Mayer, Demografiepolitik-gestalten oderverwalten?, „Aus Politikund Zeitgeschichte“ 10-11, v. 7.3.2011, S. 16. 
Wenn man nur die einheimische deutsche Bevölkerung betrachtet, liegt sie mit 1,1-1,2 noch darunter. In vielen deutschen Großstädten haben bereits $30-50 \%$ der Kinder im Kindergarten und der Grundschule einen „Migrationshintergrund“.

Im Vergleich zu Deutschland ist die demographische Situation in Polen z. Zt. noch günstiger. So werden noch mehr Kinder geboren als Menschen sterben, das Durchschnittsalter der Bevölkerung ist geringer und das Verhältnis von Jungen und Alten ist günstiger (Durchschnittsalter: Deutschland 43,7, Polen 37,5; Jugendquotient; Deutschland 20,6, Polen 21,5; Altersquotient: 30,9 zu Polen 18,9). ${ }^{3}$

Die Bevölkerungsentwicklung hat eine Vielzahl von Ursachen und Konsequenzen in Politik, Wirtschaft, auf Siedlungsräume, auf soziale Sicherungssysteme und zwar nicht nur auf die Alterssicherung, sondern auch auf das Gesundheitssystem und die Pflege, auf die Gestaltung der Zuwanderung und anderes mehr. Sie hat vielfältige gesellschaftliche Konsequenzen für Familien, Kirchen und andere Lebensbereiche. Darüber gibt es seit etwa 1980 breite gesellschaftliche Debatten ${ }^{4}$ und eine Vielzahl von Studien. Auch die katholische Kirche hat sich (teilweise zusammen mit der Ev. Kirche) in dieser Debatte zu Wort gemeldet. ${ }^{5}$

In diesem Beitrag sollen zwei der Herausforderungen näher behandelt werden. Dies sind erstens das Alterssicherungssystem und zweitens die politische Willensbildung.

\footnotetext{
3 Eurostat, Schlüsseldaten über Europa, Bonn 2010, S. 33.

4 F. X. Kaufmann, Schrumpfende Gesellschaft. Vom Bevölkerungsrückgang und seinen Folgen, Frankfurt a. M. 2005. Vgl. zur Rentenversicherung auch: J. Wiemeyer, Sozialpolitik, „Verbrauchererziehung“, Heft 19 (1982), S. 17-24.

5 Vgl. Rat der Evangelischen Kirche in Deutschland / Deutsche Bischofskonferenz, Verantwortung und Weitsicht, Erklärung zur Reform der Alterssicherung, Hannover-Bonn 2000 (Gemeinsame Texte Nr. 16); Rat der Evangelischen Kirche in Deutschland / Deutsche Bischofskonferenz, Gemeinsame Verantwortung für eine gerechte Gesellschaft (Gemeinsame Texte Nr. 22) Hannover-Bonn 2014, Familiengerechte Rente, Gutachten im Auftrag der Kommission für gesellschaftliche und soziale Fragen der Deutschen Bischofskonferenz zu einer familiengerechten Reform der gesetzlichen Rentenversicherung, Bonn 2008 (Arbeitshilfen Nr. 214); Die deutschen Bischöfe, Die Zukunft der Pflege im Alter, Ein Beitrag der katholischen Kirche, Bonn 2011 (Die deutschen Bischöfe Nr. 92).
} 


\section{Ethische Überlegungen zur Alterssicherung}

Schon der Dekalog spricht in dem vierten Gebot, „Du sollst Vater und Mutter ehren“, die Problematik der Alterssicherung an. Denn wer seine Eltern selbst gut versorgt, der kann damit rechnen, dass er selbst auch von seinen Kindern gut versorgt wird. ${ }^{6}$ So lange kein staatliches Alterssicherungssystem existiert, sind Menschen im Alter auf ihre eigenen Kinder oder eigene Ersparnisse angewiesen. Dabei sind Ersparnisse unsicher, weil eigenes Eigentum (z. B. ein Haus durch Feuer) verloren gehen kann, geraubt oder vom Staat enteignet wird, durch Inflation Geldvermögen entwertet wird usw. Kinder sind hier zuverlässiger, weil sie sich auch nach Migration, Flucht oder Vertreibung um alte Eltern kümmern. Deshalb galt Kinderlosigkeit in früheren europäischen Gesellschaften wie heute in Entwicklungsländern als schweres Schicksal, dem man ggf. auch durch Adoptionen abhelfen wollte.

In einer modernen Gesellschaft ist man aber aufgrund von zwei Elementen nicht mehr auf eigene Kinder angewiesen, so dass die individuelle und die kollektive Rationalität entkoppelt sind. Ein Rechtsstaat mit einer stabilen Währung schützt das private Eigentum, eine Sozialversicherung garantiert auch Kinderlosen eine Rente. In Deutschland gab es einen Frauenjahrgang im 20. Jahrhundert, bei dem lediglich 9\% der Frauen kinderlos waren, heute haben $24 \%$ der Frauen jüngerer Generation, vor allem Akademikerinnen mit über 30\%, keine Kinder, weitere 22,5\% nur ein Kind. ${ }^{7}$

Für ein kollektives Alterssicherungssystem ist nun entscheidend, dass genau wie in der vorindustriellen Gesellschaft und heute in Entwicklungsländern die materielle Existenz der alten Menschen nur durch die Arbeitsleistung der jüngeren Generation abgedeckt werden kann. Diese müssen einen Teil dessen, was sie erarbeiten, an die alte Generation abgeben. Konkret bedeutet das, dass das Brot, das alte Menschen essen, und die Milch, die sie trinken, immer aktuell erzeugt

6 Vgl. K. Kardinal Lehmann, Zusammenhalt und Gerechtigkeit, Solidarität und Verantwortung zwischen den Generationen, Eröffnungsreferat bei der Herbstvollversammlung der Deutschen Bischofskonferenz, Der Vorsitzende der Deutschen Bischofskonferenz Nr. 24, Bonn 2003.

7 Vgl. N. F. Schneider, J. Dorbritz, Wo bleiben die Kinder? Der niedrigen Geburtenrate auf der Spur, „Aus Politik und Zeitgeschichte“ Nr. 10-11 v. 7.3.2011, S. 26-34, hier 31. 
werden müssen, realwirtschaftlich ist Vorsorge für das Alter somit gar nicht möglich. Viele Güter (Dienstleistungen) können gar nicht gespart werden, die anderen verlieren (von Whiskey abgesehen) durch Lagerung an Wert. Wenn man nicht in Geld und Recht denkt, sondern in physischen Gütern - und das ist die reale Welt, die Welt von Geld und Recht ist eine virtuelle Welt -, gibt es keine Alternative. In der deutschen Sozialpolitik wird dies als Mackenroth-Satz bezeichnet, der 1952 betonte, dass ,aller Sozialaufwand immer aus dem Volkseinkommen der laufenden Periode gedeckt werden muß“" . Für die alte Generation stellt sich das Problem, wie sie die jüngere Generation dazu bewegen kann, einen Teil dessen, was sie erzeugt, für die Alten abzugeben. Der Generationenvertrag, nach dem die jüngere Generation jeweils für die Älteren zahlt, könnte aufgekündigt werden, was der Ökonomie-Nobelpreisträger F. A. von Hayek in drastischen Worten beschwört: „Und letzten Endes wird nicht die Moral, sondern die Tatsache, dass die Jungen die Polizei und das Militär stellen, die Frage entscheiden: Konzentrationslager für die Alten, die sich nicht selbst erhalten können, wird wahrscheinlich das Schicksal einer alten Generation sein, deren Einkommen vollständig von der Zwangsausübung auf Jüngere abhängt."

Diese Ausführungen von Hayek machen deutlich, dass es sich bei der Alterssicherung um eine wichtige ethische Fragestellung handelt. Sie betrifft das Verhältnis der Generationensolidarität bzw. der Generationengerechtigkeit ${ }^{10}$ und der Zielsetzung, die eine Gesellschaft mit einer Alterssicherung verfolgt.

Der bedeutende deutsche Sozialethiker und Jesuit Oswald von NellBreuning (1890-1991), der Verfasser der Sozialenzyklika Quadragesimo anno von Pius XII. 1931, hat immer von einem Drei-Generationen-Vertrag gesprochen. ${ }^{11}$ Dabei hat die aktive Generation zum einen die Alten zu ver-

8 G. Mackenroth, Die Reform der Sozialpolitikdurcheinen deutschen Sozialplan, in: Sozialpolitik und Sozialreform, Hrsg. von E. Boettcher, Tübingen 1957, S. 45.

$9 \quad$ F. A. v. Hayek, Verfassung der Freiburg, Tübingen 1962, S. 377.

10 Vgl. allgemein zur Generationengerechtigkeit:J. Wiemeyer, Sprawiedliwość międzypokoleniowajako wyzwanie społeczno-etyczne, ,Roczniki Nauk Społecznych“ 3 (39) 2011, S. 89-87.

11 Vgl. O. von Nell-Breuning, Soziale Sicherheit? Zu Grundfragen der Sozialordnung aus christlicher Verantwortung, Freiburg-Basel-Wien 1979, S. 75-80. 
sorgen, zum anderen eine neue nachwachsende Generation heranzuziehen. Wenn dies nicht mehr im familiären Kontext, sondern in einem Alterssicherungssystem stattfindet, hat dies für den Einzelnen durch einen Risikoausgleich in der Versicherung eine Reihe von Vorteilen. Folgende Risiken werden vermieden:

a. Ich kann eine Rente erhalten, selbst wenn wir aus medizinischen Gründen keine Kinder bekommen oder ich unverheiratet bleibe.

b. Es treten keine Konflikte mit Kindern darüber auf, wie viele Anteile ihres Einkommens sie mir abtreten sollen.

c. Ich muss nur für eine durchschnittliche Lebenserwartung der alten Menschen zahlen, nicht aber einen extremen hohen Aufwand tragen, wenn meine Eltern sehr alt werden.

d. Ich kann meinen Lebensstandard im Alter relativ absichern, und hänge nicht davon ab, ob meine Kinder vor mir sterben, wirtschaftlich erfolglos sind oder mich vernachlässigen (drogenabhängig, straffällig, arbeitslos, krank oder behindert sind, ins Ausland abwandern, ohne sich um mich zu kümmern).

Deshalb kann es für eine jüngere Generation wie für eine ältere Generation sinnvoll sein, statt innerhalb einer Familie vorzusorgen, eine Versicherung abzuschließen. Es ist daher sozialethisch sinnvoll, eine staatliche Sozialversicherung einzuführen. Der damit etablierte Generationenvertrag setzt voraus, dass die jeweils aktiv im Erwerbsleben stehende Generation immer zwei Dinge leistet, nämlich zum einen Beiträge für die Rentner zu zahlen, zum anderen eine ausreichend starke nachfolgende Generation heranzuziehen. Dieser Drei-Generationen-Zusammenhang ist in der deutschen Alterssicherung 1957 nicht etabliert worden, weil die Kindererziehung eher privat blieb, während die Versorgung der Alten überwiegend über die kollektive Versicherung lief.

Bei der Etablierung eines Alterssicherungssystems müssen folgende Grundentscheidungen getroffen werden:

a. Wie soll die Relation der Zeiten von Erwerbsarbeit und Rentenbezug sein? In Deutschland geht man von 40 Jahren Arbeit und 20 Jahren Rentenbezug aus. Wenn die Lebenserwartung um drei Jahre steigt, muss 42 Jahre gearbeitet werden und die Rentenzeit kann 21 Jahre betragen. 
b. Soll ein staatliches Alterssicherungssystem primär Armut im Alter vermeiden oder soll der im aktiven Erwerbsleben stehende ein Alterseinkommen erhalten, das in einer Relation zu seinem Lebenseinkommen steht? Wer also lange gearbeitet hat und gut verdient hat, erhält eine deutlich höhere Rente als der, der nur wenig verdient und nur relativ kurz gearbeitet hat. In diesem Konflikt zwischen Leistungs- und Bedarfsgerechtigkeit hat sich das deutsche Alterssicherungssystem auf die Seite der Leistungsgerechtigkeit (Äquivalenzprinzip) gestellt, während die Schweiz und die USA der Bedarfsgerechtigkeit Vorrang geben.

c. Soll die alte Generation am wachsenden Wohlstand der Gesellschaft teilhaben? In Deutschland wurde 1957 die dynamische Rente ${ }^{12}$ eingeführt, wobei sich die Rentenhöhe vor allem an den Lohnsteigerungen der aktiven Erwerbstätigen orientiert.

d. Wenn man ein primär an der Leistungsgerechtigkeit orientiertes System hat, muss man die Frage beantworten, wie hoch soll das Verhältnis des Einkommens eines aktiven Beschäftigten zu einem Rentner, der früher auch in seiner Einkommensklasse lag, sein. Weil bestimmte Kosten im Alter (Fahrt zur Arbeit, Berufskleidung etc.) wegfallen, strebt man in Deutschland eine Relation von 70-75\% an, die allerdings nicht allein aus der staatlichen Rentenversicherung, sondern zusätzlich durch Betriebsrenten des Arbeitgebers und durch private Ersparnisse erfolgt.

e. Weil ein Alterssicherungssystem von der Beitragszahlung und der Heranziehung einer nachfolgenden Generation abhängt, stellt sich die Frage, ob Geburt und Erziehung von Kindern in einem Alterssicherungssystem rentensteigernd berücksichtigt werden. ${ }^{13}$ In Deutschland bringt eine Geburt nach 1992 drei Jahre Anrechnung für

12 Der Bundesgeschäftsführer des Bundes der katholischen Unternehmer und spätere Professor für Sozialpolitik Wilfried Schreiben war mit seinem "Schreiber-Plan“ an der Rentenreform 1957 beteiligt. Vgl. W. Schreiber, Existenzsicherheit in der industriellen Gesellschaft, in: Sozialpolitik und Sozialreform, Hrsg. von E. Boettcher, Tübingen 1957, S. 75-114.

13 Im Gegensatz zum Schreiber-Plan wurde die Drei-Generationen-Solidarität nicht verankert, weil der damaligen Bundeskanzler Konrad Adenauer der Auffassung war, dass die „Menschen immer Kinder“ bekommen. 
die Rentenversicherung (=84 Euro Rente im Monat). Um allein durch Kinder eine Rente in Höhe der sozialen Grundsicherung zu erhalten, müsste eine Frau 8-9 Kinder haben. Für Geburten vor 1992 wurde bis 2014 ein Jahr, ab 2014 wurden 2 Jahren (28 bzw. 56 Euro) angerechnet. Wenn man Kinder höher bei der Berechnung der Renten gewichtet, würden Kinderlose relativ an Rentenhöhe verlieren.

f. Wie weit ist die Gesamtbevölkerung in ein Alterssicherungssystem einbezogen oder gibt es Gruppen mit einer eigenen Alterssicherung (Politiker, Beamte, Versorgungswerke freier Berufe), die ein besseres Leistungsniveau als in der staatlichen Versicherung erhalten? In Deutschland gehören ca. 10\% der Bevölkerung solchen begünstigten Alterssicherungssystemen an, während ein kleiner Prozentsatz gar nicht versichert ist (z. B. Ein-Personen-Unternehmer, etwa LKW-Fahrer).

Die bisherige Berücksichtigung der Kinderzahl in der Rentenversicherung hat für Deutschland keine Auswirkungen auf die Geburtenentwicklung gehabt. Damit stellt sich das Problem des Umgangs mit der Schrumpfung der Geburtenrate. Im Sinne der Generationengerechtigkeit stellt sich z. B. die Frage, wenn ich während eines Erwerbslebens 18\% meines Einkommens als Rentenbeitrag bezahlt habe, aber meine Generation für weniger Kinder gesorgt hat, darf ich dann erwarten, dass die nachfolgende Generation 25-30\% ihres Einkommens für meine Rente aufbringt?

Dies soll in zwei Schritten bedacht werden, erstens Alterssicherung in einer geschlossenen Wirtschaft (ohne Kapitalverkehr und Migration mit dem Ausland), zweitens Einbeziehung des Auslandes in die Alterssicherung.

\section{Gestaltung der Alterssicherung bei schrumpfender Bevölkerung}

\subsection{Alterssicherung in einer geschlossenen Wirtschaft}

Um die Alterssicherung in einer geschlossenen Volkswirtschaft zu gewährleisten, muss ein Zugriff der alten Generation auf das von der jüngeren Generation Erwirtschaftete bestehen. Dies kann auf vier verschiedene Arten und Weisen erfolgen: Am weitesten verbreitet ist ein 
Alterssicherungssystem, in dem Beiträge erhoben werden. Das, was die junge Generation an Beiträgen leistet, wird im nächsten Monat wieder an die Rentner ausgezahlt (Umlageverfahren). Ebenso ist es möglich, dass die Alterssicherung auf Steuermitteln basiert und aus dem laufenden Staatshaushalt bezahlt wird. Die alte Generation kann aber auch Besitztümer haben und so an jährlich aufkommenden Gewinnen, Zinsen partizipieren. Auch ist Vermögensabbau möglich, indem die Alten Besitz veräußern, Angehörige der Jungen sparen (auf Konsum verzichten), um der alten Generation etwas von ihrem Besitz abzukaufen. Es gibt nur eine ökonomisch relevante Form, mit der eine alte Generation für ihr Alter durch Sparen vorsorgen kann, nämlich wenn man Hausbesitz hat, das Haus beim Eintritt in den Ruhestand schuldenfrei und in einem guten Zustand (nicht renovierungsbedürftig) ist, dann hat man die ersparte Kaltmiete als Zusatzeinkommen. Aber auch ein Haus ist nicht problemlos, wenn z. B. eine Witwe in einem viel zu großen Haus alleine lebt und dies heizen und anderweitig unterhalten muss. Hier ist die Konstellation in Polen mit 80\% Wohneigentum günstiger als in Deutschland mit 50\%.

In Deutschland ist die Problematik groß, weil früher vier Erwerbstätige einen Rentner, in Zukunft aber - ohne Änderungen - nur noch zwei Erwerbstätige einen Rentner versorgen müssten. Dabei erhöht sich die Belastung der jungen Generation nicht nur durch ihre Schrumpfung, sondern auch durch eine immer längere Lebenserwartung der Menschen, die seit 100 Jahren praktisch Jahr für Jahr langsam ansteigt, und ein Ende der Langlebigkeit der Menschen ist nicht absehbar. Wenn man davon ausgeht, dass sich das Realeinkommen zwischen der aktiven Generation und den Rentnern in etwa parallel entwickeln soll, kann das demografische Problem weder einseitig durch Armut der Rentner noch durch drastische Beitragserhöhung für die Aktiven gelöst werden. In Deutschland wurde 1980 berechnet, dass entweder die Rentenbeiträge von 18\% des beitragspflichtigen Einkommens auf 36\% steigen müssen oder die Renten sich im Verhältnis zu den Einkommen der Aktiven halbieren müssen, so dass Altersarmut verbreitet wäre. Beide extreme Wege kommen nicht in Frage. Welche Möglichkeiten gibt es nun?

Rentenbeiträge werden in erster Linie von den aktiven Beschäftigten gezahlt. Es kann aber sein, dass nicht alle aktiven Beschäftigten (z. B. Beamte, 
Bauern, Unternehmer, Selbständige) in der Rentenversicherung sind. Wenn diese pflichtversichert werden, können zunächst die Einnahmen steigen, später können aber auch die Ansprüche wachsen. Weiterhin können mehr Einkommen einbezogen werden, wenn es z. B. eine Beitragsbemessungsgrenze gibt, nämlich dass über 5950 Euro (2014) Einkommen im Monat in Deutschland nicht mehr beitragspflichtig sind. In der Schweiz sind höhere Einkommen beitragspflichtig. Durch hohe Beiträge erwirbt man aber dort kaum Rentenansprüche, weil die Renteneinnahmen vor allem für eine Grundsicherung verwandt werden.

Es gibt nun noch zwei weitere Ansätze, für mehr Beitragseinnahmen und eine günstigere Relation zwischen Beitragszahlern und Rentnern zu sorgen. ${ }^{14}$ Der erste Ansatz betrifft die Zahl der Erwerbstätigen innerhalb der üblichen Altersgrenzen zwischen 20 und 60 Jahren. Dort geht nur ein Teil einer vollen Erwerbsarbeit nach, ein anderer Teil arbeitet nur Teilzeit, andere gehen keiner Erwerbsarbeit nach. Indem man die Zahl der Arbeitslosen, vor allem der Langzeitarbeitslosen reduziert, und andere soziale Problemfälle (Gefangene, Drogenabhängige, Obdachlose etc.) in der Gesellschaft gering hält, vergrößert man die Zahl der Erwerbstätigen. So sind pro 100000 Einwohner in Polen fast dreimal so viele Personen im Gefängnis wie in Deutschland, in den USA sogar achtmal mal so viele.

In den Arbeitsmarkt können Personen durch Rehabilitationsmaßnahmen reintegriert werden, die durch Unfälle oder Krankheiten vorzeitig erwerbsunfähig werden oder als Behinderte nicht ausreichend gefördert wurden, um einer Arbeit nachzugehen. Zahlenmäßig liegt das größte Potential bei Frauen, die Mütter und Hausfrauen sind und Teilzeit arbeiten etc. Eine stärkere Einbeziehung der Frauen in die Erwerbsarbeit darf aber in Zukunft das Problem nicht vergrößern, indem es zu einem weiteren Rückgang der Geburtenrate kommt.

Stärkere Erwerbsarbeit von Frauen und gleichzeitig Mutter zu sein, lässt sich verbinden, wenn es eine ausreichende Kinderbetreuung gibt, z. B. ab dem 1. Lebensjahr Kinderkrippen, Kindergärten und Ganztagsschulen zur Verfügung stehen. In Westdeutschland gab es ein solches System im

14 Vgl. A. Börsch-Supan, Ökonomische Auswirkungen des demografischen Wandels, „Aus Politik und Zeitgeschichte" Nr. 10-11 v. 7.3.2011, S. 19-26. 
Gegensatz zu der früheren DDR (heute ostdeutsche Bundesländer), zu Schweden und Frankreich nicht. Es wird langsam aufgebaut. In Laborem exercens (Nr. 19) wendet sich Johannes Paul II. gegen eine zu starke außerhäusliche Erwerbsarbeit der Mütter. Er fordert einen familiengerechten Lohn bzw. ein Familieneinkommen, „ohne daß die Ehefrau einem außerhäuslichen Erwerb nachgehen muß.... Einer Gesellschaft kann es nur zur Ehre gereichen, wenn sie es der Mutter ermöglicht, ..., sich der Pflege und Erziehung ihrer Kinder je nach den verschiedenen Bedürfnissen ihres Alters zu widmen."

Man muss aber in modernen, wohlhabenden Industriegesellschaften davon ausgehen, dass Frauen nicht primär aus finanziellen Gründen einer Erwerbsarbeit nachgehen. Vielmehr haben in der jüngeren Generation fast 50\% der Frauen studiert, wobei sie häufiger noch besser als Männer abschneiden, und sie wollen ihre erlernten Fähigkeiten produktiv nutzen. Während man in Schweden und Frankreich dem durch eine ausgebaute Kinderbetreuung Rechnung trägt, gehen gesellschaftliche Institutionen in Ländern wie Spanien, Italien, Österreich und Griechenland noch von einem traditionellen katholischen Familienbild aus. Dort liegt aber die Geburtenrate genauso niedrig wie in Deutschland und Polen, weil gerade gut qualifizierte Frauen dort - im Gegensatz zu Frankreich und Schweden - keine Kinder bekommen, weil es ihnen erschwert wird, gleichzeitig Mutter und erwerbstätig zu sein. In Deutschland liegt nach Umfragen die Wunschkinderzahl bei 1,7, die Geburtenrate bei 1,36. Es wäre sinnvoll, diese Diskrepanz durch geeignete Institutionen zu verringern. Eine langsamer schrumpfende Bevölkerung könnte die Probleme der Alterssicherung mildern und hätte auf der anderen Seite z. B. ökologische Vorteile.

Neben einer stärkeren Mobilisierung von Personen in der bisher üblichen Zeit der Erwerbstätigkeit besteht eine Möglichkeit darin, die Zeiten der Erwerbsarbeit zu verlängern, um so mehr Beitragszahler zu haben. Dazu kann man Schüler früher einschulen, die Dauer des Schulbesuchs bis zum Abitur verkürzen. Wenn man den Wehrdienst abschafft, bzw. die Dauer der Wehrpflicht verkürzt, stehen ebenfalls mehr Personen für den Arbeitsmarkt (statt dem ökonomisch unproduktiven Militär) zur Verfügung. Außerdem kann die tatsächliche Studiendauer reduziert werden. 
Besonders wirksam ist für ein Alterssicherungssystem, wenn das Renteneintrittsalter erhöht wird und die Menschen länger arbeiten. Eine solche Verlängerung der Lebensarbeitszeit hat nämlich einen Doppeleffekt, weil jemand, der länger arbeitet, sowohl Beiträge zahlt als auch zugleich keine Rente bezieht. Eine solche Verlängerung der Lebensarbeitszeit setzt aber voraus, dass Menschen auch nach dem bisherigen Ruhestandsalter noch leistungsfähig sind. Daher sind Anstrengungen zur Gesunderhaltung und zu einer altersgerechten Gestaltung der Arbeitsplätze notwendig. Weiterhin ist eine gute Weiterbildung der Erwerbstätigen erforderlich. Es darf in Unternehmen nicht so sein, das man Personen über 50 Jahre nicht mehr zu einer Qualifizierungsmaßnahme schickt, weil man glaubt, dass es sich nicht mehr lohnt, weil sie bald in den Ruhestand treten. Unternehmen müssen auch durch organisatorische Maßnahmen auf eine andere Alterszusammensetzung der Belegschaft reagieren, z. B. durch Entlastung Älterer von bestimmten Positionen und Aufgaben (hohe körperliche Belastung) sowie die Schaffung neuer Stellen für diese Belegschaftsgruppe, wo deren Erfahrungswissen gezielt genutzt wird. Altersgemischte Gruppen haben sich als produktiv herausgestellt. Manche Formen der Alterssicherung sind nur für gesellschaftliche Minderheiten möglich und setzen erhebliche soziale Ungleichheiten voraus, so dass sie der sozialen Gerechtigkeit widersprechen. So können sehr wohlhabende Leute mehrere Mietwohnungen besitzen und von den Mieteinnahmen leben. Da es neben Hausbesitzern, die über ihr eigenes selbst bewohntes Haus oder eine Wohnung hinaus noch Mietwohnungen besitzen, auch eigentumslose Mieterhaushalte geben muss, ist dies kein gesamtgesellschaftliches Modell.

Die bisher aufgezeigten Maßnahmen können das Problem nur mildern. Es müssen weitere Alternativen in Betracht gezogen werden, die auf eine Lastenverteilung zwischen Alten und Jungen abzielen. Konkret bedeutet dies, dass die Renten der alten Bevölkerung relativ abgesenkt werden, während die Jüngeren zugleich höhere Beiträge zahlen. Dies ist in einer wachsenden Wirtschaft möglich, weil dann niemand absolut schlechter gestellt werden muss. Wenn z. B. das Wirtschaftswachstum $2 \%$ beträgt, erhalten die junge Generation $1 \%$ davon und die ältere Generation ebenfalls 1\%, indem die Nettoeinkommen der aktiven durch 
höhere Beiträge zur Alterssicherung langsamer steigen, während die Anhebung der Renten hinter dem Wirtschaftswachstum zurückbleibt. Eine stärkere Steuerfinanzierung würde daran nichts ändern. Wenn z. B. die Mehrwertsteuer zur Rentenfinanzierung steigt, könnten Rentner weniger einkaufen. Es wäre kein Unterschied, ob man Renten weniger stark erhöht oder später durch Steuern indirekt kürzt.

Durch Ersparnisse auf der Bank kann man auch nicht für das Alter vorsorgen. Denn eine Bank oder Sparkasse muss das Geld wieder verleihen. Der größte Kreditnehmer ist der Staat. Der Staat kann entweder Zinsen für seine Schulden bedienen oder Geld in die Rentenkasse zahlen. Deshalb bringt in der Betrachtung einer geschlossenen Volkswirtschaft der Demografiefonds der Polnischen Rentenversicherung nichts für die Bewältigung der Alterung der Bevölkerung, weil er überwiegend in Staatsanleihen angelegt ist. ${ }^{15}$ Der gesamtwirtschaftliche Effekt von staatlichen Zuschüssen in die Rentenkasse oder von Zinszahlungen für Staatsanleihen eines Demografiefonds ist völlig identisch. Ähnliches gilt auch für andere kapitalgedeckte Verfahren (z. B. private Lebensversicherung, Banksparpläne etc.). Dort kann der Verteilungseffekt unterschiedlich sein, weil steuerfinanzierte (An-)teile an der Alterssicherung eher an ärmere Rentner fließen, während überdurchschnittlich Verdienende stärker sparen könnten, also die Zinsen für die Staatstitel eher an wohlhabendere Personen fließen. Andere Möglichkeiten im nationalen Kontext gibt es nicht.

Bei Geldanlagen zur Alterssicherung spielen die Zinsen eine wichtige Rolle. Wenn diese - wie gegenwärtig - sehr niedrig sind und unterhalb der Inflationsrate liegen, wird das gesparte Kapital immer weniger wert, so dass die Menschen immer weniger davon kaufen können. In Deutschland gibt es eine Zusatzversorgungskasse für die 700000 kirchlichen Mitarbeiter, die über $17 \mathrm{Mrd}$. Euro angesammelt hat, nun aber nicht mehr die notwendigen $4 \%$ Zinsen erwirtschaften kann, so dass entweder Beiträge angehoben oder Renten gesenkt werden müssen.

15 Der Demografiefonds bringt nur etwas, wenn er die inländischen Ersparnisse erhöht und der polnische Staat sich weniger im Ausland verschulden muss. 


\subsection{Alterssicherung in einer offenen Wirtschaft}

In einer offenen Wirtschaft kann man sich zur Stabilisierung der Alterssicherung zweier Mechanismen bedienen, nämlich zum einen der Wanderung von Arbeitskräften, zum anderen der Kapitalbildung im Ausland. Wie bereits erwähnt hat Deutschland sein Geburtendefizit seit über 40 Jahren durch laufende Zuwanderung aus dem Ausland ausgeglichen. ${ }^{16}$ In den nächsten Jahrzehnten rechnet man damit, dass netto (Zuzüge minus Abzüge) mindestens 200000 Personen jährlich zuwandern, die den Sterbeüberschuss ausgleichen. Dieser Sterbeüberschuss kann aber auch auf 400-500 000 steigen, so dass eine stärkere Zuwanderung notwendig wird. Hier kann nicht die ganze Zuwanderungsproblematik diskutiert werden. Es sei nur darauf hingewiesen, dass Zuwanderung für die Alterssicherung nur funktioniert, wenn es sich um qualifizierte Arbeitskräfte handelt, die bereit sind, in Deutschland hohe Steuern und Sozialabgaben zu zahlen. Möglicherweise werden solche qualifizierten Arbeitskräfte aber in Länder wie die USA auswandern, wo die Steuerlast geringer ist.

Offene Grenzen und Probleme der Alterssicherung können auch einen umgekehrten Effekt haben: Wenn zur Sicherung der Rente die Steuern und Abgaben zu hoch werden, könnten Angehörige der jüngeren Generation sagen, ich bin noch bereit, für meine Eltern zu sorgen, nicht aber für Personen zu zahlen, die es versäumt haben, eigene Kinder zu bekommen. Man wandert also ins Ausland aus, nimmt seine alten Eltern mit oder versorgt diese mit Geldüberweisungen aus dem Ausland, während wegen der Auswanderung qualifizierter Kräfte im Inland die Wirtschaft immer weiter schrumpft und der Regierung nichts übrig bleibt, als die Renten fortlaufend zu kürzen. Damit könnte eine Situation eintreten, die einer vorindustriellen Gesellschaft entspricht, wo Kinderlose in Armut leben und fast bis zu ihrem Tod arbeiten mussten. Ein Land, das zur Sicherung seiner Altersversorgung auf Zuwanderung setzt, muss seine qualifizierte Bevölkerung im Inland halten. Dies wird umso schwieriger, weil vie-

16 Vgl. D. von Löffelholz, Demografischer Wandel und Migration als Megatrends, „Aus Politik und Zeitgeschichte" Nr. 10-11, v. 7. 3.2011, S. 34-42. 
le junge Leute gute Sprachkenntnisse haben und bereits (z. B. bei einem Auslandstudium) längere Zeit im Ausland verbracht haben. Wenn man ausländische Studenten z. B. durch englischsprachige Studiengänge anzieht, von denen ein Teil im Inland bleibt, kann eine Strategie gelingen.

Ein Problem in der EU mit ihrem großen Einkommensgefälle kann sein, dass die Hocheinkommensländer Migranten aus anderen EU-Ländern anlocken, was diesen - wenn man qualifizierte Kräfte (die dort teuer ausgebildet wurden) anwirbt - einen kurzfristigen Vorteil bringt. Es ist aber problematisch, wenn durch die Anwerbung besonders qualifizierter Arbeitskräfte in anderen Ländern wichtige Zweige wie z. B. (durch die Abwerbung von Ärzten und Pflegepersonal) das Gesundheitswesen gefährdet und die wirtschaftliche Entwicklung gehemmt wird. Dann werden die kurzfristigen Gewinner durch innereuropäische Zahlungen (Strukturfonds) mehr Transfers an die ärmeren EU-Länder zahlen müssen.

Neben Menschen kann man auch das Kapital bei offenen Grenzen einbeziehen. Deutschland hat seit mehr als 10 Jahren hohe Exportüberschüsse. Weil es mehr Güter im Ausland verkauft als es von dort erwirbt, sammelt Deutschland immer mehr Vermögen im Ausland an. In Deutschland wird dies auch mit der demographischen Entwicklung begründet. Denn wenn nach 2020 die geburtenstarken Jahrgänge in den Ruhestand treten, kann dieses Vermögen wieder abgebaut werden, indem Deutschland mehr Güter (zur Versorgung der Alten) importiert als es in die Welt hinaus exportiert. Ein solcher zeitweiser Kapitalexport kann für die beteiligten Länder zum gegenseitigen Vorteil sein. Hat ein Land eine jüngere Bevölkerung und strömen geburtenstarke Jahrgänge auf den Arbeitsmarkt, benötigt diese Gesellschaft Kapital, um zusätzliche Arbeitsplätze zu schaffen. Wenn durch richtige Investitionen dort die Wirtschaft wächst, können später aus dem hohen Wachstum die Zinsen gezahlt und das Kapital zurückgezahlt werden.

Eine solche Auslandstrategie setzt zunächst einen Exportüberschuss voraus. Polen hat in den letzten 20 Jahren fast immer ein Leistungsbilanzdefizit gehabt, also Schulden gegenüber dem Ausland angehäuft. Auch wenn man wie Deutschland Überschüsse hat, gibt es doch eine Vielzahl von Risiken. Das erste Risiko bei Auslandsanlagen ist, dass es außerhalb des Währungsraumes des EURO ein Währungsrisiko gibt. Wenn die andere 
Währung, in der man zeitweise Geld investiert hat, im Wert sinkt, kann die Rechnung nicht aufgehen. Weitere Risiken liegen in politischen, gesellschaftlichen und ökonomischen Entwicklungen im Ausland. Es können in Länder, in denen man investiert hat, Regierungen an die Macht kommen, die ökonomisch ein Land ruinieren. In einem Land können soziale Unruhen oder gar Bürgerkriege auftreten. Weiterhin ist denkbar, dass ein Land in Kriege verwickelt wird oder aber von größeren Naturkatastrophen betroffen wird. Da es sich bei Kapitalanlagen zur Alterssicherung im Ausland um Prozesse handelt, bei denen man in Zeiträumen von 15-30 Jahren denken muss, sind solche Entwicklungen schwer vorhersehbar. Wenn man die Kapitalanlagen zur Risikostreuung auf verschiedene Länder aufteilt, kann auch eine weltweite Wirtschaftskrise auftreten, wie die internationale Finanzkrise 2008, die einen Teil der deutschen Kapitalanlagen im Ausland entwertet hat. So hatte Deutschland wertvolle Maschinen und Autos in die USA geliefert und sich im Gegenzug dafür - wie sich später herausgestellt hat - weitgehend wertlose amerikanische Finanzpapiere geben lassen.

Wenn man eine solche Strategie im EURO-Raum betreibt, ist zwar das Währungsrisiko nicht gegeben, es sei denn ein Land scheidet aus dem EURO-Raum aus. Wie der Schuldenschnitt Griechenlands zeigt, sind aber nicht alle Anlagen in anderen EU-Ländern sicher. So hatte etwa das Land Nordrhein-Westfalen einen Teil des Geldes für seine Ruhestandsbeamten in griechische Staatsanleihen angelegt, was bei dem griechischen Schuldenschnitt verloren ging. Andererseits werden im EURO-Raum bestimmte Risiken (wie Kriege) unwahrscheinlicher sein. Allerdings fehlen in der EU weitgehend die demographischen Voraussetzungen für eine Kapitalexportstrategie, weil in fast allen EU-Ländern die Geburtenrate mehr oder weniger stark unterhalb der Sicherung der Bevölkerungszahl liegt.

\subsection{Fazit}

Jede Gestaltung der Alterssicherung eines Landes muss von der nicht änderbaren Tatsache ausgehen, dass Sozialaufwand zur Sicherung der alten Bevölkerung immer aus dem laufenden Sozialprodukt bestritten werden muss und dass es keine andere Quelle in realwirtschaftlicher Hinsicht 
gibt. Da man nur Güter, nicht aber Geld essen kann, ist die realwirtschaftliche Seite entscheidend. Ob es sich rechtlich um Versorgungsansprüche von Beamten handelt, ob es um Ansprüche gegen eine staatliche Rentenversicherung geht, ob man Ersparnisse auf der Bank oder eine Lebensversicherung hat, dies alles sind rechtliche Fiktionen, die jeweils an die realwirtschaftlichen Bedingungen angepasst werden müssen, indem z. B. der Staat Renten kürzt, auf Ersparnisse oder Lebensversicherungen eine neue oder höhere Steuer erhebt.

Die bisher diskutierten Vorschläge beseitigen aber nicht das Grundproblem der Divergenz zwischen der individuellen Rationalität (Kinder benötigt man für die eigene Alterssicherung nicht) und der kollektiven Rationalität (die Gesamtgesellschaft benötigt eine hinreichend stark nachwachsende Generation). Man könnte beides damit wieder stärker zusammenbringen, indem nur Personen mit zwei oder mehr Kindern die volle Rente erhalten, während die Rente für Personen mit einem Kind oder keinen Kindern deutlich gekürzt würde. Was kinderlose oder kinderarme an Aufwendungen für die eigenen Kinder sparen, könnten sie dann nicht konsumieren, sondern müssten es kapitalbildend (einschließlich der Risiken z. B. für Auslandsanlagen) investieren. ${ }^{17}$

Für Polen ist relevant, dass seine wirtschaftliche Produktivität weiter steigen muss. Damit kann es längerfristig auch zu einem Exportüberschuss kommen, so dass zumindest in einer demographisch ungünstigen Situation nicht noch das weniger Produzierte zum Teil an das Ausland zur Begleichung seiner Auslandsschulden abgegeben werden muss. Weiterhin bleiben dann mehr Polen im Land selbst und wandern nicht weiter ins Ausland ab. Länder, die ein hohes Pro-Kopf-Einkommen aufweisen, ziehen Zuwanderer an. Da alle europäischen Länder (einschließlich Russland) tendenziell eine schrumpfende Bevölkerung aufweisen, können aber Zuwanderer nach Europa in größerer Zahl nur aus dem Nahen Osten, aus Afrika südlich der Sahara sowie aus Asien kommen. Daher stellt sich die Frage der gesellschaftlichen Akzeptanz von Zuwanderern aus fremden Kulturen.

17 Vgl. J. Wiemeyer, Gerechtigkeit zwischen Generationen als wirtschaftsethisches Problem, „Ethica“ 12 (2004) 1, S. 71-94. 


\section{Die politischen Konsequenzen einer alternden Bevölkerung}

In der öffentlichen Diskussion über die Konsequenzen einer älter werdenden Bevölkerung wird die Frage diskutiert, ob die in den oben skizzierten Abschnitten genannten Mechanismen tatsächlich politisch durchsetzbar sind, vor allem, wenn sie nicht rechtzeitig eingeleitet und beschlossen werden. Droht einer Gesellschaft eine „Gerontokratie“, also eine Herrschaft der Alten? ${ }^{18}$ Denn wenn erst einmal die Hälfte der Wähler ein Alter von 60 Jahren und älter erreicht hat, könnte in einer Demokratie eine alte Generation ihre Interessen zu Lasten der jüngeren Generation egoistisch durchsetzen.

Dies kann sich erstens darauf beziehen, dass die ältere Generation relative Rentenkürzungen nicht hinnimmt, sondern höhere Beitragslasten von der jüngeren Generation zur Sicherung ihrer Renten fordert. Zweitens können rentennahe Jahrgänge sich einer Anhebung des Renteneintrittsalters verweigern, indem sie politische Mehrheiten dagegen organisieren. Die Belastungen für die Alterssicherung können in einer prosperierenden Wirtschaft leichter getragen werden. Dies setzt aber Strukturveränderungen voraus, etwa Investitionen in die öffentliche Infrastruktur und anderes mehr. Alte Jahrgänge könnten sich aber z. B. größeren Bauprojekten (Flughäfen, Bahnhöfen, Straßen- und Schienenwegen etc.) verweigern, die zwar Eingriffe in die Natur darstellen, aber ökonomisch langfristig notwendig erscheinen. Ebenso könnten sie sich gegen neue Techniken wenden, die möglicherweise Risiken darstellen.

Besonders große Vorbehalte kann eine relativ alte Bevölkerung aufweisen, wenn es eine größere Anzahl jüngerer Zuwanderer gibt, die etwa wegen ihres äußeren Aussehens und Verhaltens sichtbar in Erscheinung treten.

Zwar denkt eine ältere Generation, die Kinder und Enkel hat, häufig auch an nachwachsenden Generationen, was vor allem auch dann deutlich wird, wenn z. B. Großeltern ihre studierenden Enkel durch Zuwendungen im Studium unterstützen, bei der Kinderbetreuung helfen oder ander-

18 Vgl. B. Munismus, Ältere Taktgeber in der alternden Gesellschaft?, „Aus Politik und Zeitgeschichte" Nr. 4-5- v. 21.1.2013, S. 57-62. 
weitig einspringen. Da aber das demographische Problem auch dadurch verursacht wird, dass viele Ältere keine Kinder und Enkel mehr haben, könnte sich eher eine selbstbezogene Verhaltensweise durchsetzen.

Die politische Einflussnahme der alten Menschen kann über verschiedene Kanäle laufen. In Deutschland ist es ein Phänomen, dass die Mitgliedschaft in den politischen Parteien (CDU / CSU und SPD) ein hohes Durchschnittsalter aufweist. Damit beeinflussen sie die Programmatik der Parteien wie die Aufstellung von Kandidaten. Weiterhin hat die ältere Bevölkerung die höchste Wahlbeteiligung. Drittens sind sie für Politiker etablierter Parteien die berechenbarste Wählergruppe, indem sie vielfach treue Stammwähler sind. Hingegen wählen Jüngere eher neue Parteien, wie die Piraten, die aber, weil sie nicht ins Parlament kommen, politisch irrelevant werden.

Für die Bundestagswahlen in Deutschland lässt sich hinsichtlich der Wahlbeteiligung festhalten ${ }^{19}$, dass über 60-Jährige teilweise eine 15-20\% höhere Wahlbeteiligung (80\% zu 60-65\%) haben als die unter 30-Jährigen. Erst bei den ganz Alten lässt wegen gesundheitlicher Gebrechen die Wahlbeteiligung nach.

Die größte Partei (CDU / CSU) hat ihre Stammwähler in der alten Bevölkerung. Bei den über 60-jährigen Wählern insgesamt erzielt sie 49\%, bei den Frauen sogar ihr absolut bestes Ergebnis mit 53\%, bei den unter 30-Jährigen nur 34\%, bei Studenten und Auszubildenden 30\%, (insgesamt 41\%). ${ }^{20}$ Den älteren Wählerinnen haben die CDU / CSU einen Rentenzuschlag von 28 Euro pro Kind und Monat versprochen. Ebenso hat die Sozialdemokratie den höchsten Wähleranteil bei den über 60-Jährigen. Diese sollen mit 63 Jahren ohne Abschlag in den Ruhestand gehen können. Für Deutschland ist aber offen, ob die Tatsache, dass mehr als 50\% der Alten CDU/ CSU wählen, darauf zurückzuführen ist, dass Menschen im Alter (generell) konservativer werden oder dass die ältere Generation stärker christlich geprägt ist und ein Wahlverhalten, dass sie bereits

19 Vgl. B. Munimus, Ältere Taktgeber in der alternden Gesellschaft?, „Aus Politik und Zeitgeschichte“ Nr. 4-5 v. 21.1.2013, S. 59.

${ }_{20}$ Vgl. M. Jung, Y. Schroth, A. Wolf, Angela Merkels Sieg in der Mitte, „Aus Politik und Zeitgeschichte" Nr. 48-49 v. 25.11.2013, S. 9-20, hier 15. 
vor mehreren Jahrzehnten erlernt hat, fortführt. Wenn Letzteres zutrifft, müsste die Dominanz der Unionsparteien in der alten Generation schwinden, weil nun die von den Studentenunruhen von 1968 geprägten Personen in den Ruhestand eintreten.

Weiterhin ist festzustellen, dass je geringer der soziale Status ist, desto weniger Personen zur Wahl gehen. Auch dieser Effekt kann soziale Ungleichheit verstärken. Die Befürchtung einer „Gerontokratie“ erscheint aber übertrieben, weil die Interessenslage auch alter Menschen heterogen ist, so dass sich keine gemeinsamen politischen Forderungen älterer Menschen, z. B. reicheren bzw. ärmeren Älteren ergeben. ${ }^{21}$ Daher wird eine reine Rentnerpartei nicht erfolgreich sein, so dass es in den großen Volksparteien immer Kompromisse zwischen Älteren und Jüngeren geben muss.

\section{Schlussbemerkung}

Angesichts der demographischen Entwicklung in Deutschland wie in Polen ist es verfehlt, die Entwicklung zu dramatisieren und von „demografischer Katastrophe“ zu sprechen oder eine „Diktatur der Alten“ zu befürchten. Vielmehr kann eine langfristig angelegte Politik, die sich um eine leistungsfähige Wirtschaft bemüht, dafür sorgen, dass Menschen länger gesund bleiben, sich weiterqualifizieren und länger arbeiten sowie eine gesteuerte Zuwanderung miteinbeziehen, so dass die Probleme zu bewältigen sind, selbst wenn es nicht gelingen sollte, die Geburtenziffer anzuheben. So treten in Deutschland nur geringe Probleme auf, wenn es in Zukunft gelingt, so viele Personen in die Erwerbsarbeit zu integrieren, wie dies gegenwärtig bereits in Dänemark der Fall ist. ${ }^{22}$ Natürlich wäre es wünschenswert, wenn es in beiden Ländern zu einem Anstieg der

${ }_{21}$ Vgl. S. Wurm, F. Berner, C. Tesch-Römer, Altersbilder im Wandel, „Aus Politik und Zeitgeschichte“, Nr. 4-5 v. 21.1.2013, S. 3-8.

22 Vgl. A. Bösch-Supan, Ökonomische Auswirkungen des demografischen Wandels, „Aus Politik und Zeitgeschichte" Nr. 10-11 v. 7.3.2011, S. 23-25. 
Geburtenzahlen kommen sollte. Weiter kann eine gewisse Kapitalbildung im Ausland etwas zur Problemmilderung beitragen.

Zusammenfassung: Der Artikel diskutiert die Konsequenzen des Bevölkerungsrückgangs in Deutschland und anderen europäischen Staat, speziell für die Alterssicherung. Einkommen alter Menschen muss Teil des nationalen Einkommens derselben Periode sein, so dass es durch Abgaben von der aktiven Arbeitsbevölkerung gewonnen werden kann. Kapitalbildung lediglich außerhalb der nationalen Grenzen kann nur geringe Hilfe bringen, ist aber auch mit vielen Risiken verbunden. Die politischen Konsequenzen einer länger lebenden Bevölkerung und des Geburtenrückgangs ist, dass Personen über 60 Jahre die Mehrheit der Wähler darstellen. Es besteht aber keine Gefahr der Herrschaft der Alten, weil die Interessen alter Menschen heterogen sind. 


\section{Summary}

Security for the aging people and the politic in the decreasing population

The article discusses the consequences of birth decline in Germany and other European countries especially for the old-age pension system. Income of old people has to be part of the national income of the same period so that it could be gained through taxes from the active working population. Capital building outside the national borders only can bring little help but it is connected with many risks. The political implication of longer living population and birth decline is that the majority of voters are older than 60 years. But there is no fear of government of the aging people because the interests of old people are heterogeneous.

Keywords: elderly people, pastoral care, Church and the senior citizens

\section{Alterssicherung und Politik bei schrumpfender Bevölkerung}

Durch sinkende Geburtenzahlen und eine steigende Lebenserwartung gerade die Alterssicherungssysteme in Deutschland wie in Polen in finanzielle Schwierigkeiten. Die Probleme sind aber lösbar, wenn die Lebensarbeitszeit verlängert wird, mehr Personen im Erwerbsalter (z. B. Arbeitslose, Frauen) einer Beschäftigung nachgehen und es eine gewisse Zuwanderung von qualifizierten Erwerbspersonen aus dem Ausland gibt. Da die Interessen der älteren Generation heterogen sind, werden notwendige Reformen nicht durch eine Dominanz der Wähler im Rentenalter verhindert.

Schlüsselbegriffe: Generationengerechtigkeit, Demographische Entwicklung,

Alterssicherung, Migration

\section{Zabezpieczenie osób starszych i polityka w kurczącym się społeczeństwie}

Artykuł omawia konsekwencje malejącej liczby urodzin w Niemczech i innych krajach europejskich, zwłaszcza w relacji do systemu emerytalnego. Dochody osób starszych muszą być częścią dochodu krajowego w tym samym czasie, w którym jest on pobierany z podatków od ludności czynnej zawodowo. Dochody z kapitału lokowanego za granicą mogą pomóc tylko w niewielkim stopniu, a obarczone są znacznym ryzykiem. Godna odnotowania jest polityczna konsekwencja wydłużania się życia i obniżającej się liczby narodzin w społeczeństwie - większość wyborców ma ponad sześćdziesiąt lat. Rządzący nie muszą obawiać się osób starszych, bo ich dochody są zróżnicowane.

Słowa kluczowe: ludzie starsi, opieka duszpasterska, Kościół i starsi 


\section{Bibliografie}

Börsch-Supan A., Ökonomische Auswirkungen des demografischen Wandels, „Aus Politik und Zeitgeschichte" Nr. 10-11 v. 7.3.2011, S. 19-26.

Die deutschen Bischöfe, Die Zukunft der Pflege im Alter, Ein Beitrag der katholischen Kirche, Bonn 2011 (Die Deutschen Bischöfe Nr. 92).

Eurostat, Schlüsseldaten über Europa, Bonn 2010.

Familiengerechte Rente, Gutachten im Auftrag der Kommission für gesellschaftliche und soziale Fragen der Deutschen Bischofskonferenz zu einer familiengerechten Reform der gesetzlichen Rentenversicherung (Arbeitshilfen Nr. 214), Bonn 2008.

Hayek F. A. v., Verfassung der Freiburg, Tübingen 1962.

Jung M., Schroth Y., Wolf A., Angela Merkels Sieg in der Mitte, „Aus Politik und Zeitgeschichte“ Nr. 48-49 v. 25.11.2013, S. 9-20.

Kaufmann F. X., Schrumpfende Gesellschaft. Vom Bevölkerungsrückgang und seinen Folgen, Frankfurt a. M. 2005.

Lehmann K. Kardinal, Zusammenhalt und Gerechtigkeit, Solidarität und Verantwortung zwischen den Generationen, Eröffnungsreferat bei der Herbstvollversammlung der Deutschen Bischofskonferenz, Der Vorsitzende der Deutschen Bischofskonferenz Nr. 24, Bonn 2003.

Löffelholz D. v., Demografischer Wandel und Migration als Megatrends, „Aus Politik und Zeitgeschichte" Nr. 10-11, v. 7. 3.2011, S. 34-42.

Mackenroth G., Ein deutscher Sozialplan, in: Sozialpolitik und Sozialreform, Hrsg. v. E. Boettcher, Tübingen 1956.

Mayer T., Demografiepolitik - gestalten oder verwalten, „Aus Politik und Zeitgeschichte“ 10-11, v. 7.3.2011, S. 16.

Munismus B., Ältere Taktgeber in der alternden Gesellschaft?, „Aus Politik und Zeitgeschichte“ Nr. 4-5 v. 21.1.2013, S. 57-62.

Nell-Breuning O. v., Soziale Sicherheit? Zu Grundfragen der Sozialordnung aus christlicher Verantwortung, Freiburg-Basel-Wien 1979, S. 75-80.

Schneider N. F., Dorbritz J., Wo bleiben die Kinder? Der niedrigen Geburtenrate auf der Spur, „Aus Politik und Zeitgeschichte“ Nr. 10-11 v. 7.3.2011, S. 26-34.

Rat der Evangelischen Kirche in Deutschland, Deutsche Bischofskonferenz, Verantwortung und Weitsicht, Erklärung zur Reform der Alterssicherung, Hannover-Bonn 2000 (Gemeinsame Texte Nr. 16).

Rat der Evangelischen Kirche in Deutschland, Deutsche Bischofskonferenz, Gemeinsame Verantwortung für eine gerechte Gesellschaft, Hannover-Bonn 2014 (Gemeinsame Texte Nr. 22).

Wiemeyer J., Sozialpolitik, „Verbrauchererziehung“ Heft 19 (1982), S. 17-24,

Wiemeyer J., Sprawiedliwość międzypokoleniowa jako wyzwanie społeczno-etyczne, „Roczniki Nauk Społecznych” 3 (39) 2011, S. 89-87.

Wiemeyer J., Gerechtigkeit zwischen Generationen als wirtschaftsethisches Problem, „Ethica“ 12 (2004), S. 71-94.

Wurm S., Berner F., Tesch-Römer C., Altersbilder im Wandel, „Aus Politik und Zeitgeschichte" Nr. 4-5 v. 21.1.2013, S. 3-8. 